\title{
Cancer-Specific Loss of $\beta$-Defensin 1 in Renal and Prostatic Carcinomas
}

\author{
Carlton D. Donald, Carrie Q. Sun, So Dug Lim, Jill Macoska, Cynthia Cohen, \\ Mahul B. Amin, Andrew N. Young, Tomas A. Ganz, Fray F. Marshall, and \\ John A. Petros
}

Winship Cancer Institute (CDD, CQS, MBA, FFM, JAP), Department of Urology (CDD, CQS, MBA, FFM, JAP), and Department of Pathology (SDL, CC, MBA, ANY, JAP), Emory University School of Medicine, and Atlanta Veterans Administration Medical Center (ANY, JAP), Atlanta, Georgia; Department of Urology (JM), University of Michigan, Ann Arbor, Michigan; and Department of Medicine and Will Rogers Institute for Pulmonary Research (TAG), University of California, Los Angeles School of Medicine, Los Angeles, California

SUMMARY: In a previous large-scale gene expression profiling study of renal epithelial neoplasms, human $\beta$-defensin-1 (DEFB1) was found to be significantly down-regulated in conventional clear cell (renal) carcinoma. We have now completed an expanded expression analysis of this gene. We performed immunohistochemical analysis for the DEFB1 protein in clinical specimens of both renal cell carcinoma and prostate cancer. In a subset of prostate cancers, we performed laser capture microdissection and RT-PCR to correlate mRNA levels with protein levels. Overall, $82 \%$ of prostate cancers exhibit either complete loss of protein expression or only minimal expression, whereas the adjacent benign epithelium retained expression in all cases. Similarly, $90 \%$ of renal cell carcinomas show cancer-specific loss of DEFB1 protein. In the prostate cancer subset analysis, mRNA levels correlate with protein levels. We have thus demonstrated the cancer-specific down-regulation of DEFB1 in a large sample of prostatic and renal carcinomas and validated one of the key findings of previous cancer gene profiling studies of prostatic and renal neoplasia. (Lab Invest 2003, 83:501-505).

$T$ o further define our previously reported results of large-scale gene expression profiling in renal cell carcinoma (Young et al, 2001), we have performed an expanded protein and RNA expression analysis for one of the genes that showed the greatest cancerspecific down-regulation. In that analysis, conventional (clear cell) renal cell carcinoma had (on average) a 7 -fold lower expression of the human $\beta$-defensin-1 (DEFB1) gene than benign renal tissue (Young et al, 2001). In addition, the chromosomal location of $D E F B 1$ (8p23) is within the smallest overlapping region of deletion defined in a loss of heterozygosity study performed on 96 conventional renal cell carcinomas (Schullerus et al, 1999). Finally, DEFB1 plays an important role in the innate and adaptive immune response at epithelial surfaces (Yang et al, 1999) and is cytotoxic to mammalian cells in culture (Zucht et al, 1998), suggesting a possible role in preventing or eliminating tumors. Thus, it is possible that loss of this protein enhances or promotes tumor formation.

DOI: 10.1097/01.LAB.0000063929.61760.F6

Received November 4, 2002.

Address reprint requests to: Dr. J. A. Petros, 1365-B Clifton Road, Room 4222, Atlanta, Georgia 30322.E-mail: jpetros@emory.edu

\section{Results}

\section{Immunohistochemistry (IHC)}

Renal. Forty-eight conventional renal cell carcinomas were assayed for DEFB1 protein expression by IHC. Normal renal tubules and collecting ducts were uniformly positive, whereas areas of carcinoma varied widely in their expression of DEFB1 protein (Fig. 1A). Of 48 cancers tested, $33(69 \%)$ demonstrated no DEFB1 protein expression and $10(21 \%)$ showed only weak staining, with less than $10 \%$ of cells being positive. Moderate staining was noted in $10 \%$ of renal cancers (Fig. 2B).

Prostate. In an exactly analogous manner, 100 prostatic adenocarcinoma specimens were assayed for DEFB1 expression by IHC. Only sections containing defined regions of normal prostate, which represented an internal positive control, were analyzed. DEFB1 was universally detected in normal prostate tissue but frequently was lost in prostatic intraepithelial neoplasm (Fig. 1B) and prostate cancer (Fig. 1C). All of the normal prostate sections exhibited strong DEFB1 staining. However, DEFB1 expression varied widely in the malignant regions. Overall, $82 \%$ of the cancer sections analyzed did not stain or stained only weakly for DEFB1 protein. Furthermore, of the 100 cases analyzed, 39 exhibited no DEFB1 protein expression in prostate cancer. Fourteen tumors had moderate 

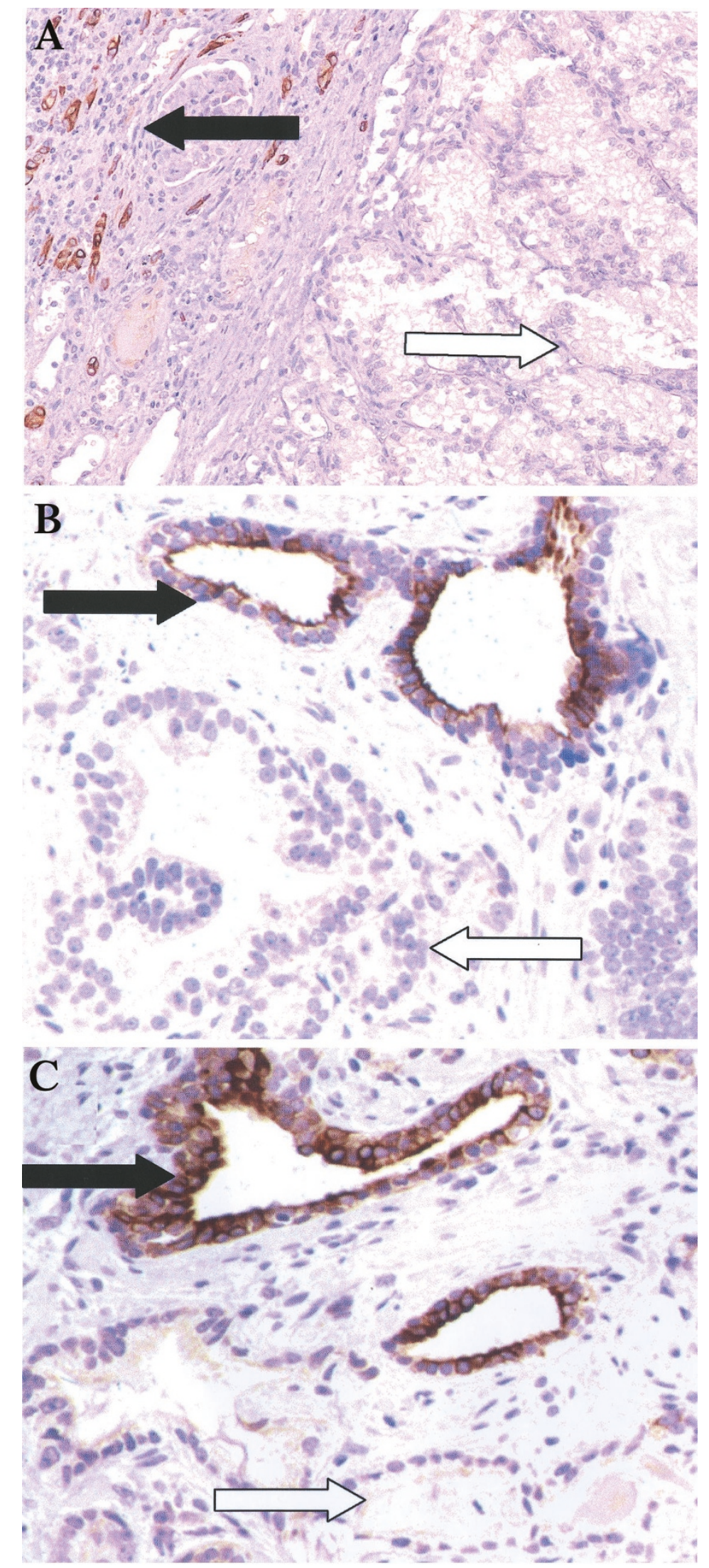

\section{Figure 1.}

Immunohistochemical analysis of $\beta$-defensin-1 (DEFB1) in the kidney and prostate. A, Normal kidney expresses DEFB1 (black arrow), whereas adjacent kidney cancer does not (white arrow). B, Prostatic intraepithelial neoplasm showing loss of DEFB1 expression (white arrow). Here, DEFB1 is shown in a normal gland (black arrow). C, Malignant prostate tissue showing absence of DEFB1 (white arrow). Black arrow, normal glands positive for DEFB1. 

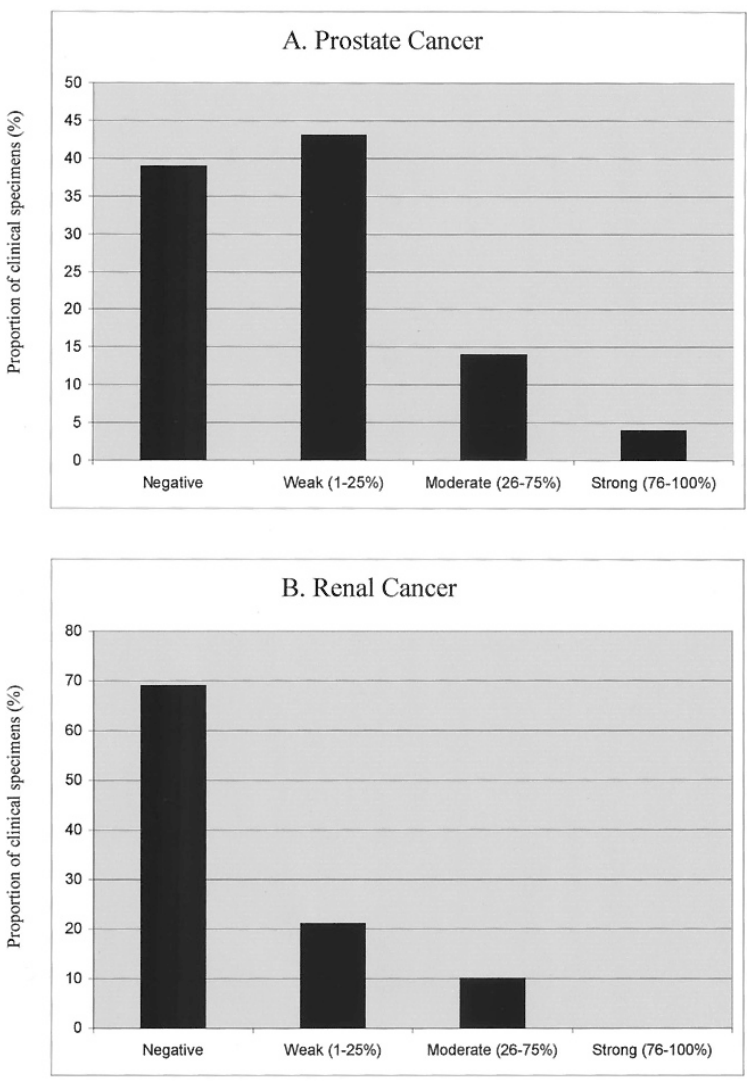

Figure 2.

Proportion of clinical specimens with detectable DEFB1 protein in renal and prostate cancers. A, Prostate cancer $(n=100)$; B, renal cancer $(n=48)$. In each graph the results of immunohistochemistry staining intensity are as assigned by two urologic pathologists. Each individual case was assigned one of four staining intensities based on the percentage of malignant cells that stained positively for DEFB1: Negative $=$ no positive cells; Weak $=1 \%$ to $25 \%$; Moderate $=26 \%$ to $75 \%$; and Strong $=76 \%$ to $100 \%$ of malignant epithelial cells positive for DEFB1 protein expression. The $y$-axis indicates the proportion of cases with that staining intensity.

staining (26-75\%) and only the remaining four exhibited strong staining (>76\%) (Fig. 2A).

\section{Comparison of DEFB1 mRNA and Protein Expression}

To determine whether loss of protein expression was a result of decreased mRNA levels, we compared DEFB1 protein expression with mRNA transcript levels using quantitative RT-PCR in 12 cases randomly chosen from the 100 primary prostate specimens. This subset analysis allowed us to compare mRNA and protein levels from adjacent benign and malignant prostatic epithelium from the same individual. Eight of the 12 cases demonstrated decreased levels of DEFB1 protein in cancer compared with the benign epithelium, and all 8 also had lower transcript levels compared with normal tissue. Two cases of the subset of 12 exhibited more DEFB1 in cancer, with the same difference seen at the message level. The remaining two cases demonstrated relatively equal levels of DEFB1 protein in normal and malignant tissues, but one exhibited slightly more DEFB1 message levels in cancer and the other seemed to have slightly less message in cancer. In summary, 10 of 12 specimens analyzed for both protein and mRNA expression demonstrated concordant cancer-associated alterations of $D E F B 1$ gene expression, with 8 showing decreased levels in the cancer and 2 showing increased expression. The remaining two cases had similar expression in the cancer and normal areas at the protein level and only minor differences at the mRNA level.

\section{Discussion}

Using cDNA microarray gene expression profiling we have previously identified DEFB1 as one of the most significantly down-regulated transcripts in conventional renal cell carcinoma (Young et al, 2001). Because the DEFB1 gene is located in an area of chromosome $8 p$ frequently deleted in both renal and prostatic carcinomas, we determined whether expression of this gene was altered in these two malignancies. We have demonstrated that human DEFB1 expression is decreased in the malignant epithelial cells of a significant majority of clinical samples of human renal cell and prostatic carcinomas. We have found that although the adjacent benign epithelium is universally positive for DEFB1 expression in both the kidney and prostate, histologically identifiable malignant cells have lost protein expression in $67 \%$ of the prostate cancers and $90 \%$ of the renal cell carcinomas. In a subset of prostate cancer cases that were subjected to laser capture microdissection and real-time quantitative RT-PCR, a cancer-specific loss of DEFB1 mRNA was found that corresponded with immunohistochemical analysis showing loss of protein expression. We therefore conclude that the observed cancerspecific loss of expression is a result of either decreased transcription of the DEFB1 gene or increased degradation of this transcript as opposed to posttranslational regulation. Although these observations clearly document the frequent cancer-specific loss of this protein, our study does not address whether the loss of DEFB1 causes or enhances transformation or is simply a secondary phenomenon reflecting the loss of differentiation that occurs in many cancers. In this regard, the existing literature offers little about the physiologic role of DEFB1 or its possible role in tumorigenesis.

As early as 1994, the cytotoxic activity of the structurally related $\alpha$-defensins was noted (Kagan et al, 1995). Although these data were generated with the $\alpha$-defensins, their structural similarity to the $\beta$-defensins makes a similar functionality possible, even though untested. DEFB1 is a member of a large family of defensin genes located on the short arm of chromosome 8 . Members of this family encode small $(4-7 \mathrm{kDa})$ peptides that are either found in leukocytes ( $\alpha$-defensins) or secreted at epithelial surfaces $(\beta$ defensins) and function primarily as naturally occurring antimicrobial peptides. DEFB1 (a $\beta$-defensin) is somewhat anomalous in this regard. DEFB1 has only weak antimicrobial activity, and this activity is suppressed in solutions containing physiologic sodium chloride concentrations. Furthermore, in contrast to its 
weak antimicrobial activity, DEFB1 has a strong cytotoxic potential toward mammalian cells, leading to speculation that DEFB1 had functions other than the antimicrobial activity so characteristic of other defensins (Zucht et al, 1998). Similarly, speculation about a nonantimicrobial function of DEFB1 followed the demonstration of DEFB1 mRNA in the developing kidney by in situ hybridization. The expression of DEFB1 early in development suggests that it has physiologic functions rather than only host microbial defense. No such functionality has yet been definitively assigned to this peptide.

Both DEFB1 and DEFB2 are potent "microchemokines" that attract immature dendritic cells and memory $\mathrm{T}$ cells via specific interaction with the chemokine receptor CCR6 (Yang et al, 1999). It is through this action that $\beta$-defensins are thought to play an important role in both innate and adaptive immune responses (Yang et al, 1999).

Considering the existing literature together with the data presented in this article, it is interesting to speculate that DEFB1 may contribute to host antitumor immunity or otherwise function as a tumor suppressor gene. If DEFB1 expression is lost at some point during the malignant transformation, the host may be less likely to recognize the tumor as "foreign," perhaps through a failure of dendritic cells and memory T cells to be recruited to newly transformed epithelial cells, and the tumor may be more likely to survive. Although our data do not address these functional questions, it is also of interest to note that the DEFB1 gene is located in an area of chromosome 8 that is purported to contain multiple tumor suppressor genes.

Chromosome $8 p$ frequently undergoes DNA sequence copy number loss in human neoplasms. Because of the high frequency of loss of $8 p$ in different tumors, this chromosome is implicated as being the location for one or more tumor suppressor genes or DNA repair genes that may be involved in the pathogenesis of these tumors (Knutila et al, 1999). In fact, loss of heterozygosity studies have identified three distinct areas of loss on $8 p$, suggesting the existence of at least three separate tumor suppressor genes.

Prostate cancer has been extensively characterized for chromosome $8 p$ loss. In 1999, analysis of 60 tumors revealed distinct loci of loss at 8 p23, 8p22, and 8p12 (Perinchery et al, 1999). Although functional data have been generally lacking, complementation studies using whole human chromosome 8 introduced into a highly metastatic rat prostate cancer showed that human chromosome 8 was capable of suppressing metastasis and that the 8 p23 region was specifically included in the region identified as containing the metastasis-suppressing activity (Ichikawa et al, 1996). This region contains the DEFB1 gene.

In summary, we present data showing the cancerspecific loss of human DEFB1 expression in human clinical samples of prostatic and renal carcinomas. The DEFB1 gene lies within one of the regions of the short arm of chromosome 8 purported to contain one or more tumor suppressor genes. DEFB1 protein normally serves as an important component of the innate and adaptive immune response because of its ability to interact with a specific chemokine receptor on dendritic cells and memory $\mathrm{T}$ cells. In addition, DEFB1 has direct cytotoxic activity toward mammalian cells. The loss of expression of this protein by transformed epithelium may therefore inhibit host immune recognition or defensin-mediated tumor cell cytotoxicity, although this was not specifically tested in this study.

\section{Materials and Methods}

\section{Tissue Samples}

Tissue acquisition was performed in accordance with an institutional review board-approved protocol, including processing, sectioning, histologic characterization, DNA purification, and PCR amplification. The immunohistochemical staining was performed retrospectively on fixed tissues from radical prostatectomies and radical nephrectomies recently performed at Emory University Hospital. One hundred radical prostatectomy specimens that contained both benign and malignant epithelial cells were used for immunohistochemical analysis. Forty-eight radical nephrectomy specimens were similarly used for routine immunohistochemical staining. In 12 of the radical prostatectomy specimens, frozen sections were prospectively obtained for laser capture microdissection, RNA preparation, and real-time RT-PCR from both benign and malignant epithelium. For quantitative RT-PCR analysis, mRNA was isolated from histologic slides using the National Institutes of Health recommended protocol with slight modification. laser capture microdissection was then performed to ensure that tissue samples assayed consisted of pure populations of tumor and normal cells.

\section{Immunohistochemical Analysis of DEFB1 Expression}

IHC analysis of paraffin-embedded tissue sections was accomplished after dewaxing and pretreatment by microwaving at $100^{\circ} \mathrm{C}$ in Tris EDTA buffer for antigen retrieval, then incubation with rabbit polyclonal antiserum antibody (developed by TAG) for 1 hour at 1:800 dilution (Valore et al, 1998). After a 25-minute incubation period, sections were washed and treated with commercial biotinylated second antiIg, followed by avidin coupled to biotinylated horseradish peroxidase. The immunohistochemical reactions were visualized using the brown peroxidase substrate diaminobenzidine and counterstained with hematoxylin. Staining of epithelial cytoplasm was graded by two pathologists into four groups: absent (0), weak (1), moderate (2), or strong (3) staining.

\section{Quantitative Real-Time RT-PCR}

We performed quantitative real-time RT-PCR on laser capture microdissection-generated tumor/normal pairs for 12 randomly chosen cases of the 100 primary prostate tumors to compare DEFB1 expression in benign and malignant epithelium from the same individual. Two-step 
QRT-PCR was performed on cDNA generated using the MultiScribe Reverse Transcriptase from the TaqMan Reverse Transcription System and the SYBR Green PCR Master Mix (PE Biosystems). The primer pairs 5'GTTGCCTGCCAGTCGCCATGAGAACTTCCTAC-3' and 5'-TGGCCTTCCCTCTGTA ACAGGTGCCTTGAATT-3' were generated from the published DEFB-1 sequence. Reactions were performed in a MicroAmp Optical 96-well Reaction Plate (PE Biosystems). Forty cycles of PCR were performed under standard conditions using an annealing temperature of $60^{\circ} \mathrm{C}$. Quantification was determined by mean fluorescence intensity at the cycle number where exponential amplification began (threshold cycle) and was averaged from the values obtained from duplicate repeats. There was an inverse relationship between message level and threshold cycle number. In addition, $\beta$-actin was used as a housekeeping gene to normalize the initial content of total cDNA. DEFB1 expression was calculated as the relative expression ratio between DEFB1 and $\beta$-actin and was compared in benign and malignant prostate epithelium for each case. All reactions were performed in duplicate or triplicate.

\section{References}

Ichikawa T, Nihei N, Kuramochi H, Kawana Y, Killary AM, Rinker-Schaeffer CW, Barrett JC, Isaacs JT, Kugoh H, Oshimura M, and Shimazaki J (1996). Metastasis suppressor genes for prostate cancer. Prostate Suppl 6:31-35.

Kagan J, Stein J, Babaian RJ, Joe YS, Pisters LL, Glassman $A B$, von Eschenbach AC, and Troncoso P (1995). Homozygous deletions at 8 p22 and $8 p 21$ in prostate cancer implicate these regions as the sites for candidate tumor suppressor genes. Oncogene 11:2121-2126.
Knutila S, Aalto Y, Autio K, Bjorkqvist AM, El-Rifai W, Hemmer S, Huhta T, Kettunen E, Kiuru-Duhlefelt S, Larramendy ML, Lushnikova T, Monni O, Pere H, Tapper J, Tarkkanen M, Varis A, Wasenius VM, Wolf $M$, and Zhu $Y$ (1999). DNA copy number losses in human neoplasms. Am J Pathol 155:683-694.

Perinchery G, Bukurov N, Nakajima K, Chang J, Hooda M, Oh BR, and Dahiya R (1999). Loss of two new loci on chromosome 8 (8p23 and 8q12-13) in human prostate cancer. Int J Oncol 14:495-500.

Schullerus D, von Knobloch R, Chudek J, Herbers J, and Kovacs $G$ (1999). Microsatellite analysis reveals deletion of a large region at chromosome $8 p$ in conventional renal cell carcinoma. Int J Cancer 80:22-24.

Valore EV, Park CH, Quayle AJ, Wiles KR, McCray PB Jr, and Ganz T (1998). Human beta-defensin-1: An antimicrobial peptide of urogenital tissues. J Clin Invest 101:1633-1642.

Yang D, Chertov O, Bykovskaia SN, Chen Q, Buffo MJ, Shogan J, Anderson M, Schroder JM, Wang JM, Howard OMZ, and Oppenheim JJ (1999). $\beta$-defensins: Linking innate and adaptive immunity through dendritic and T cell CCR6. Science 286:525-528.

Young AN, Amin MB, Moreno CS, Lim SD, Cohen C, Petros JA, Marshall FF, and Neish AS (2001). Expression profiling of renal epithelial neoplasms: A method for tumor classification and discovery of diagnostic molecular markers. Am J Pathol 158:1639-1651.

Zucht HD, Grabowsky J, Schrader M, Liepke C, Jurgens M, Schulz-Knappe P, and Forssmann WG (1998). Human betadefensin-1: A urinary peptide present in variant molecular forms and its putative functional implication. Eur J Med Res 20:315-323. 